\title{
X-shooter spectroscopy of FU Tauri A ${ }^{\star}$
}

\author{
B. Stelzer ${ }^{1}$, J. M. Alcalá ${ }^{2}$, A. Scholz ${ }^{3}$, A. Natta ${ }^{3,4}$, S. Randich ${ }^{4}$, and E. Covino ${ }^{2}$ \\ 1 INAF - Osservatorio Astronomico di Palermo, Piazza del Parlamento 1, 90134 Palermo, Italy \\ e-mail: stelzer@astropa.inaf.it \\ 2 INAF - Osservatorio Astronomico di Capodimonte, Via Moiariello 16, 80131 Napoli, Italy \\ 3 School of Cosmic Physics, Dublin Institute for Advanced Studies, 31 Fitzwilliam Place, Dublin 2, Ireland \\ ${ }^{4}$ INAF - Osservatorio Astrofisico di Arcetri, Large E. Fermi 5, 50125 Firenze, Italy
}

Received 15 November 2012 / Accepted 19 December 2012

\begin{abstract}
We have analyzed a broad-band optical and near-infrared spectrum of FU Tau A, which is a presumed young brown dwarf in the Taurus star forming region that has intrigued both theorists and observers by its overluminosity in the Hertzsprung-Russell (HR) diagram with respect to standard pre-main sequence evolutionary models. FU Tau A is brighter than any other Taurus member of the same or similar spectral type, and various phenomena (accretion, activity, binarity) have been put forth as possible explanations. The new data, obtained with the X-shooter spectrograph at the Very Large Telescope, include an unprecedented wealth of information on stellar parameters and simultaneously observed accretion and outflow indicators for FU Tau A. We present the first measurements of gravity $(\log g=3.5 \pm 0.5)$, radial velocity $\left(R V=22.5 \pm 2.9 \mathrm{~km} \mathrm{~s}^{-1}\right)$, rotational velocity $\left(v \sin i=20 \pm 5 \mathrm{~km} \mathrm{~s}^{-1}\right)$, and lithium equivalent width $\left(E W_{\mathrm{Li}}=430 \pm 20 \mathrm{~m} \AA\right)$ for FU Tau A. From the rotational velocity and the published period we infer a disk inclination of $i \gtrsim 50^{\circ}$. The lithium content is much lower than theoretically expected for such a young very low-mass object, adding another puzzling feature to this object's properties. We determine the mass accretion rate of FU Tau A from comparison of the luminosities of 20 emission lines to empirical calibrations from the literature and find a mean of $\log \dot{M}_{\text {acc }}\left[M_{\odot} / \mathrm{yr}\right]=-9.9$ with standard deviation $\sigma=0.2$. The accretion rate determined independently from modeling of the excess emission in the Balmer and Paschen continua is consistent with this value. The corresponding accretion luminosity is too low to make a significant contribution to the bolometric luminosity. Strong magnetic activity affecting the stellar parameters or binarity of FU Tau A, both combined with extreme youth, may be responsible for its position in the HR diagram. The existence of an outflow in FU Tau A is demonstrated through the first detection of forbidden emission lines from which we obtain an estimate for the mass loss rate, $\log \dot{M}_{\text {out }}\left[M_{\odot} / \mathrm{yr}\right]<-10.4$. The mass outflow and inflow rates can be combined to yield $\dot{M}_{\text {out }} / \dot{M}_{\text {acc }} \sim 0.3$, a value that is in agreement with jet-launching models.
\end{abstract}

Key words. brown dwarfs - stars: pre-main sequence - accretion, accretion disks - stars: individual: FU Tau A

\section{Introduction}

FU Tau A was discovered by Luhman et al. (2009a) as the primary component of a young brown dwarf (BD) binary in the Barnard 215 cloud. This dark cloud is considered to be part of the Taurus molecular cloud complex, so a distance of $140 \mathrm{pc}$ has been assigned (e.g. Torres et al. 2009). According to midinfrared images from Spitzer, only one other young star, FT Tau, is present within $0.5^{\circ}$ of FU Tau (Luhman et al. 2009b). Its isolated position makes FU Tau a benchmark object for studies of brown dwarf formation scenarios since most mechanisms, such as ejection from a protostellar cluster, photo-evaporation, or disk fragmentation require the presence of higher mass stars (see e.g. Whitworth et al. 2007).

Luhman et al. (2009a) have obtained optical and near-IR low-resolution spectroscopy for the primary (FU Tau A) and an optical spectrum for the secondary. They derived spectral types of M7.25 and M9.25 for the two components, respectively. By comparison to spectral templates, they extracted different values for the extinction from the optical $\left(A_{\mathrm{V}} \sim 2 \mathrm{mag}\right)$ and the near-IR $\left(A_{\mathrm{V}}<1 \mathrm{mag}\right)$ spectra of the primary, FU Tau A, and adopted the higher value for calculating its bolometric luminosity. Masses of $0.05 M_{\odot}$ and $0.015 M_{\odot}$ were inferred when comparing the

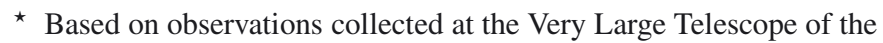
European Southern Observatory under program 086.C-0173(A). position of the two objects in the Hertzsprung-Russell (HR) diagram to the evolutionary pre-main sequence models of Baraffe et al. (1998) and Chabrier et al. (2000). However, as noted by Luhman et al. (2009a), in the HR diagram FU Tau A is located well above the youngest (1 Myr) isochrone of these models. The secondary, FU Tau B, also seems younger than 1 Myr albeit by a lesser amount, contesting the general notion of coevality for the components in binaries (Luhman et al. 2010).

In this article, we aim at investigating the nature of the primary, FU Tau A. Various signatures of youth are apparent in the available observations of this object. The presence of a circumstellar disk was established from an analysis of its spectral energy distribution (SED), where excess emission over a photosphere of the same spectral type is observed in all four Spitzer/IRAC bands, i.e. at $3.6 \mu \mathrm{m}, 4.5 \mu \mathrm{m}, 5.8 \mu \mathrm{m}$, and $8.0 \mu \mathrm{m}$ (Luhman et al. 2009a). Similarly, an excess in the blue part of the SED indicates ongoing accretion. This is bolstered by the high equivalent width (EW) of $\mathrm{H} \alpha$ emission measured in the Luhman et al. low-resolution spectra (93 $\mathrm{A})$, as well as the large width of the $\mathrm{H} \alpha$ profile in a medium-resolution spectrum from Gemini analyzed by Stelzer et al. (2010). The full-width at $10 \%$ of the peak height was measured to be $350 \mathrm{~km} \mathrm{~s}^{-1}$, distinctly higher than the canonical limit of $200 \mathrm{~km} \mathrm{~s}^{-1}$ considered to represent the borderline between magnetic activity and accretion-dominated $\mathrm{H} \alpha$ emission in BDs (Jayawardhana et al. 2003). Applying the calibration provided by Natta et al. (2004) to the $\mathrm{H} \alpha 10 \%$ width 
yielded a mass accretion rate of $3.5 \times 10^{-10} M_{\odot} / y r$. Stelzer et al. (2010) caution that the $\mathrm{H} \alpha$ line is only marginally resolved in the Gemini spectrum. However, the mass accretion rate derived from the line flux of the He I $\lambda 587.6 \mathrm{~nm}$ line in the same spectrum, using the calibration of Herczeg \& Hillenbrand (2008) is about a factor two higher than the value obtained from $\mathrm{H} \alpha$, providing further evidence for strong accretion.

Recently, the FU Tau binary has been the target of a Chandra $\mathrm{X}$-ray observation with the aim of studying magnetic activity in two coeval BDs of slightly different effective temperatures and masses. Unexpectedly, while the secondary was not detected, the primary showed very strong and soft X-ray emission, reminiscent of the T Tauri star TW Hya where the bulk of X-rays is produced in accretion shocks rather than the stellar corona (e.g. Kastner et al. 2002). Considering the atypically low X-ray temperature and this analogy with TW Hya, FU Tau A may be the first BD where X-ray emission from accretion shocks has been detected (Stelzer et al. 2010).

The current observational picture of FU Tau presents a number of ambiguities. First, the over-luminosity in the HR diagram, especially for the primary, allows for different interpretations as discussed by Stelzer et al. (2010) and Scholz et al. (2012). It could be due to extreme youth, to the primary being an unresolved binary, or to a strong contribution from accretion to the luminosity of FU Tau A. Alternatively, the inhibiting influence of magnetic fields and/or rotation onto convection might lead to lower effective temperature for a given luminosity and, consequently, a wrong mass estimate (Chabrier et al. 2007). Indeed, the modulations in photometric time series reveal the presence of both hot spots, i.e. accretion, and cool spots, i.e. magnetic activity (Scholz et al. 2012). Secondly, the velocities derived in the $\mathrm{H} \alpha$ profile are lower than the infall speeds suggested by the observed X-ray temperature, leading to a doubt about how to interpret the origin of the X-ray emission in accretion shocks.

Aiming at a better understanding of this puzzling BD, we have obtained broad-band spectroscopy from the UV to the nearIR and multi-color time-series photometry for FU Tau A. The motivation for collecting these data was to seek for a better understanding of its accretion and activity characteristics as possible causes of the overluminosity of FU Tau A in the HR diagram through the study of spectral signatures. The new photometry is part of our study of FU Tau A's long-term variability. The observations and data analysis are described in Sect. 2. Stellar properties are derived in Sect. 3. In Sects. 4 and 5 we examine the outflow and accretion characteristics of FU Tau A, and in Sect. 6 we discuss our results, and Sect. 7 presents a summary.

\section{Observations and data reduction}

FU Tau A was observed on Jan. 11, 2011 with the X-shooter spectrograph at the Very Large Telescope (VLT). The data were acquired within the INAF/GTO time (Alcalá et al. 2011). With its three spectrograph arms, X-shooter provides simultaneous wavelength coverage from 300-2480 nm. Slit widths of $1.0^{\prime \prime} / 0.9^{\prime \prime} / 0.9^{\prime \prime}$ were used in the UVB/VIS/NIR arms, respectively, yielding spectral resolutions of 5100/8800/5600. The total exposure time in each of the three spectrograph arms was $1800 \mathrm{~s}$. We obtained a signal-to-noise ratio of $1-8$ in the UVB, $10-20$ in the VIS, and $20-30$ in the NIR arm. The data were obtained in nod mode and were reduced independently for each arm with the X-shooter pipeline, v1.3.7 (Modigliani et al. 2010). Following the standard steps including bias or dark subtraction, flat-fielding, optimal extraction, wavelength calibration, sky subtraction, correction for atmospheric extinction, and flux calibration. However, the pipeline flux-calibrated spectra are not corrected for telluric absorption bands. The telluric correction was performed independently in the VIS and NIR spectra in the following way. For the VIS arm, the spectrum of the telluric standard (HIP 20789, spectral type B7 V), normalized to the continuum, was used as input in the IRAF" ${ }^{1}$ task "telluric". For the NIR spectrum, a response function was first derived by dividing the non-flux-calibrated spectrum of the telluric by a blackbody of the same effective temperature $\left(T_{\text {eff }}=13000 \mathrm{~K}\right)$ as the telluric standard. This response function, containing the telluric lines, was then used as input in the IRAF task "telluric". In this way, the telluric correction and the correction for the response function were done simultaneously. Although the shape of the resulting spectrum after this procedure is correct, the flux calibration is only relative to the response function. To bring the NIR spectrum into an absolute flux scale we multiplied it by a factor that was estimated using the pipeline flux-calibrated spectrum. Finally, wavelength shifts due to instrumental flexures were corrected using the flexcomp package within the pipeline. The precision of the wavelength calibration is better than 0.01 pix corresponding to $0.002 \mathrm{~nm}$ in the UVB and VIS arms and to $0.006 \mathrm{~nm}$ in the NIR arm.

The flux-calibrated spectrum of FUTauA for the full $\mathrm{X}$-shooter wavelength range is shown in Fig. 1. Photometry from the literature is overlaid, demonstrating the high quality of the flux calibration. In particular, the match of the different instrumental arms is very good. In fact, during the observation the seeing was excellent $\left(\approx 0.85^{\prime \prime}\right)$.

\section{Stellar properties}

\subsection{Spectral type and extinction}

We estimated the spectral type and extinction of FU Tau A by comparing it to the spectral templates defined by Manara et al. (2013, henceforth MTR13) on the basis of X-shooter spectra for 24 nonaccreting and unabsorbed young stellar objects (YSOs) in various star forming regions. These spectra define a continuous spectral sequence from M0 to M6.5 with steps of 0.5 in the spectral subclass. In addition they include two objects at the end of the M subclass. At their young ages (1-10 Myr), these objects are expected to have similar gravity to FU Tau A. We determined the spectral type and the extinction of FU Tau A simultaneously by artificially reddening the templates between $A_{\mathrm{V}}=0 . .2$ mag until the best match to FU Tau A was found. For the reddening we used the extinction law of Weingartner \& Draine (2001). In Fig. 2, the spectrum of FU Tau A is compared to that of Par-Lup3-1 (M6.5) and DENIS-P J124514.1442907 (M9). The spectrum of Par-Lup3-1 appears very similar to FU Tau A, and we conclude that the spectral type of FU Tau A is M6.5 or slightly later. The gap in the spectral type sequence of MTR13 does not allow us to put stronger constraints. We estimated an extinction of $A_{\mathrm{V}} \sim 0.5 \pm 0.5 \mathrm{mag}$. The templates in Fig. 2 are both reddened by this amount.

In a second approach to determining the spectral type of FU Tau A, we calculated various spectral indices following Riddick et al. (2007) for optical wavelengths, the $\mathrm{H}_{2} \mathrm{O}$ index defined by Allers et al. (2007), and the $\mathrm{H}_{2} \mathrm{O}-\mathrm{K} 2$ index from Rojas-Ayala et al. (2012) for the near-IR spectrum. The indices that we used are consistent with the spectral sequence of

1 IRAF is distributed by the National Optical Astronomy Observatories, which are operated by the Association of Universities for Research in Astronomy, Inc., under cooperative agreement with the National Science Foundation. 


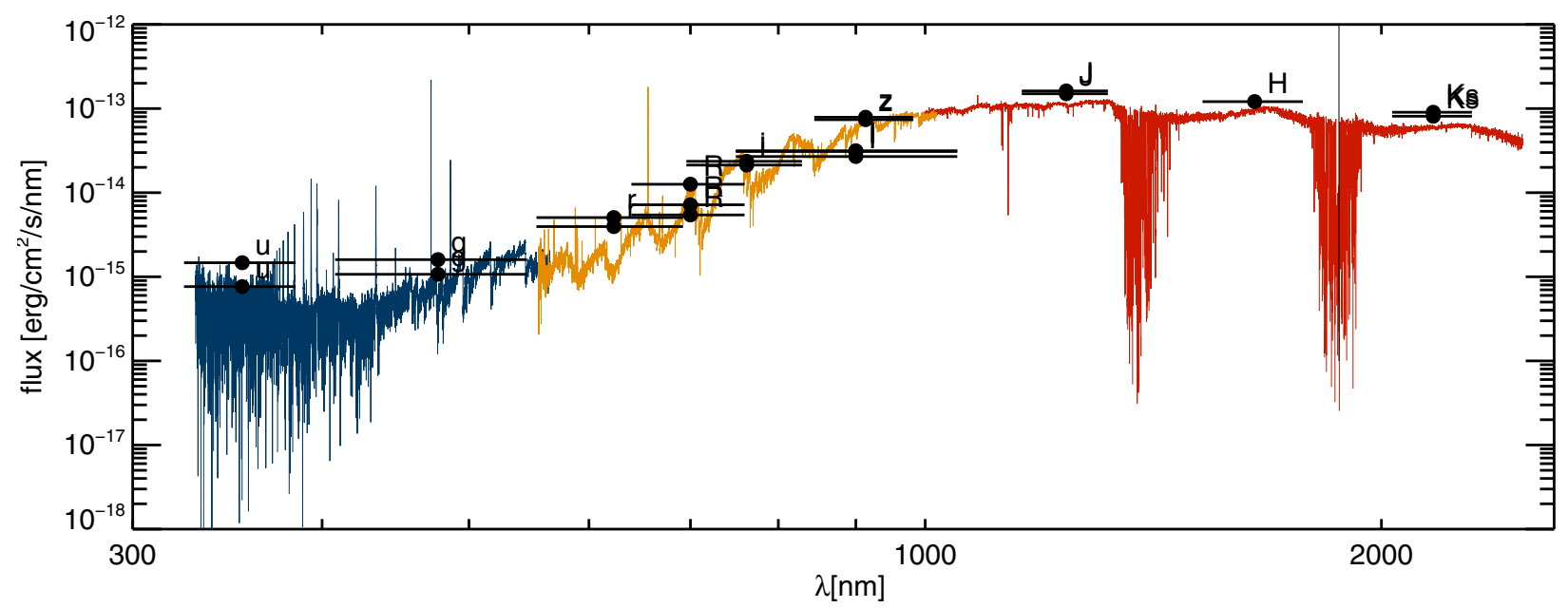

Fig. 1. Broad-band flux-calibrated X-shooter spectrum of FU Tau A. Published photometry from Luhman et al. (2009a), from Scholz et al. (2012) and unpublished RIJK photometry obtained in September 2012 with Andicam at the $1.3 \mathrm{~m}$ telescope on Cerro Tololo as part of the SMARTS program DUBLIN-12A-001 is overplotted.

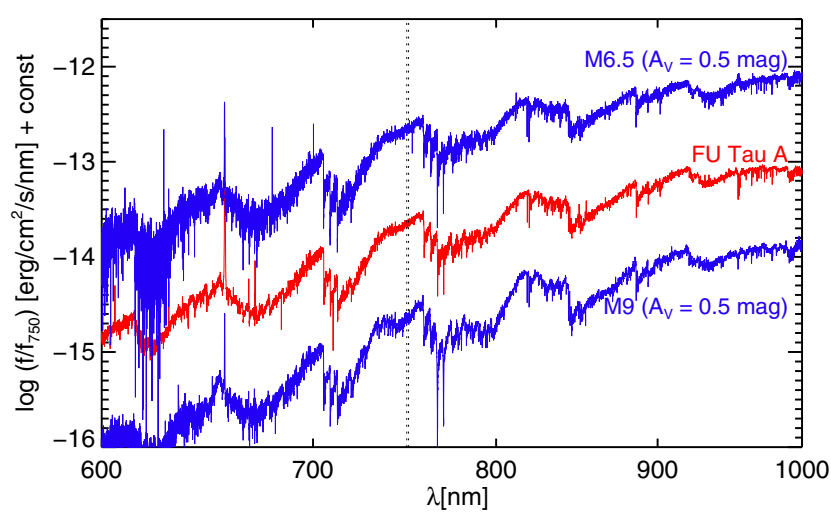

Fig. 2. X-shooter VIS spectrum of FU Tau A compared to two spectral templates from MTR13. A reddening of $A_{\mathrm{V}}=0.5 \mathrm{mag}$ has been applied to the templates. The spectra are normalized at $750 \mathrm{~nm}$, and the templates are vertically offset.

MTR13. The flux ratios for the spectral indices were calculated from the spectrum of FU Tau A after modifying it by various amounts of extinction between $A_{\mathrm{V}}=0$ and 2 mag. We confirm that the optical spectral indices from Riddick et al. (2007) are independent of extinction for the range of extinctions tested here. We derived a spectral type of M6.6 \pm 0.4 for FU Tau A, building the average and standard deviation of the VO2, R1, R2, R3, TiO8465, and VO7445 indices. The $\mathrm{H}_{2} \mathrm{O}$ index yields $\mathrm{M} 6.7 \pm$ 0.2 and the $\mathrm{H}_{2} \mathrm{O}-\mathrm{K} 2$ index M7.1 for a range of $A_{\mathrm{V}}=0 \ldots 1 \mathrm{mag}$. Riddick et al. (2007) and Allers et al. (2007) provide calibrations specifically for young $\mathrm{M}$ dwarfs; i.e., their spectral indices are independent of gravity, and we find excellent correspondence in the derived spectral types. The slightly later spectral type obtained from the $\mathrm{H}_{2} \mathrm{O}-\mathrm{K} 2$ index may be related to the fact that Rojas-Ayala et al. (2012) have calibrated it for nearby (evolved) M dwarfs.

The spectral type we obtained from the X-shooter spectrum with the two methods explained above is also consistent with our earlier results from low-resolution spectroscopy where we found values between M6.6 and M6.8 in a series of five spectra (Scholz et al. 2012). Using a different set of spectral templates Luhman et al. (2009a) find a similar, slightly cooler spectral type of M7.25 \pm 0.25 .
The Luhman et al. value for the extinction derived from their optical spectrum $\left(A_{\mathrm{V}}<1 \mathrm{mag}\right)$ is also similar to ours, but they find a higher value from the near-IR spectrum $\left(A_{\mathrm{V}}=2 \mathrm{mag}\right)$. The latter value is clearly incompatible with the $\mathrm{X}$-shooter NIR spectrum for which we derive $A_{\mathrm{V}} \sim 0.75 \pm 0.5 \mathrm{mag}$ in an analogous way as the one described for the VIS. Throughout this paper we adopt the spectral type and extinction derived from the VIS spectrum. We expect the determination of extinction using zeroextinction spectral templates observed with the same instrument to be preciser in the VIS than in the NIR because the effects of extinction are generally greater in the VIS than in the NIR.

\subsection{Stellar parameters}

Our new measures for the spectral type and the extinction can be used to revise the stellar parameters of FU Tau A. The radius of FU Tau A can be obtained from Stefan-Boltzmann's law. Rather than use the literature value for the bolometric luminosity, we recompute it by considering the difference in our adopted $A_{\mathrm{V}}$ and the value assumed by Luhman et al. (2009a). From the $J$ magnitude given by Luhman et al. (2009a) and a bolometric correction of B.C.J $=2.05$ (Dahn et al. 2002), we find $L_{\mathrm{bol}}=0.13 L_{\odot}$. We measured a slightly earlier spectral type than Luhman et al. (2009a), yielding an effective temperature of $T_{\text {eff }}=2940 \mathrm{~K}$ for a temperature scale midway between dwarfs and giants (Luhman 1999). For these numbers we derive $R_{*}=1.4 R_{\odot}$, which is substantially lower than the value resulting from the luminosity and temperature given by Luhman et al. (2009a), $R_{*}=1.8 R_{\odot}$. After extrapolating the position of FU Tau A in the HR diagram down to the youngest isochrone of the evolutionary models by Chabrier et al. (2000), the mass is $0.08 M_{\odot}$, placing FU Tau A right at the hydrogen-burning mass limit.

We used the gravity- and temperature-sensitive absorption doublets of $\mathrm{Na}$ I at $\lambda \lambda 818.33,819.48 \mathrm{~nm}$ and of $\mathrm{K} \mathrm{I}$ at $\lambda \lambda 766.48$, $769.89 \mathrm{~nm}$ to determine $\log g$ by comparing the X-shooter data to synthetic spectra. We retrieved BT-DUSTY model spectra for a range of $T_{\text {eff }}$ around the expected value of $2940 \mathrm{~K}$ and a range of $\log g$ values from the star, brown dwarf $\mathcal{E}$ planet atmosphere web simulator (Allard et al. 2012) which is electronically available ${ }^{2}$. In Fig. 3 the synthetic spectra for $T_{\text {eff }}=2900 \mathrm{~K}$ with three different values for $\log g$ are overlaid on the spectrum of

2 http://phoenix.ens-lyon.fr/simulator/index.faces 


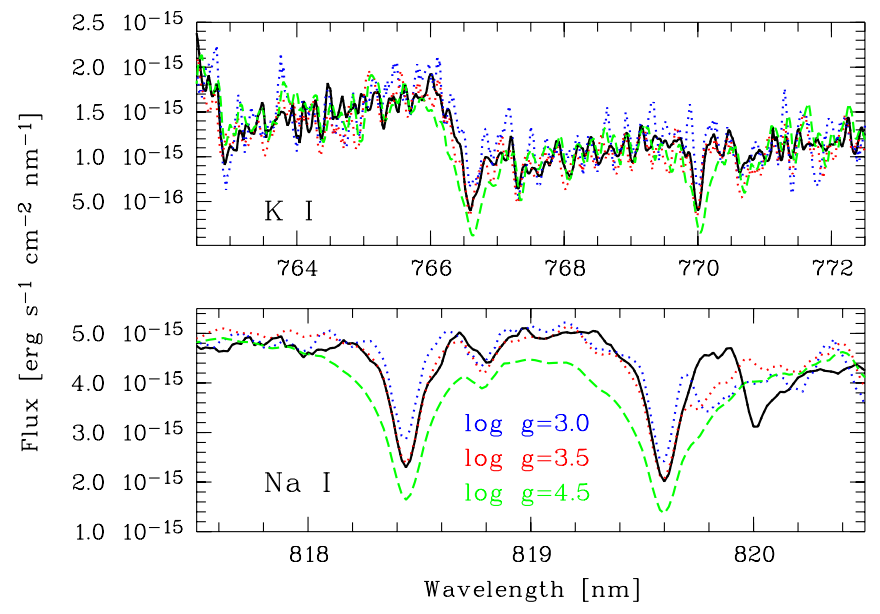

Fig. 3. Detail of the spectrum of FU Tau A (black solid lines) in the wavelength range around the K I (upper panel) and $\mathrm{Na}$ I (lower panel) absorption doublets. The spectrum of FU Tau A has been corrected for telluric absorption lines. The blue and the red dotted lines and the green dashed line represent synthetic spectra with $\log g$ as labeled for $T_{\text {eff }}=$ $2900 \mathrm{~K}$ and rotationally broadened to $v \sin i=20 \mathrm{~km} \mathrm{~s}^{-1}$.

FU Tau A in the region of the $\mathrm{Na}$ and $\mathrm{K}$ doublets. The model spectra have been rotationally broadened to $v \sin i=20 \mathrm{~km} \mathrm{~s}^{-1}$, the rotation rate determined for FU Tau A in Sect. 3.5. The width of the observed lines in Fig. 3 is in good agreement with a gravity of $\log g=3.5 \pm 0.5$. The uncertainty is due to the 0.5 steps for $\log g$ in the grid of synthetic spectra. The gravity derived from the spectrum agrees with the value expected from the evolutionary models of Baraffe et al. (1998), adopting the stellar parameters derived above and an age of 1 Myr.

\subsection{Lithium absorption}

Theoretical models predict that the lithium content of low-mass stars is rapidly depleted throughout the first $\sim 50 \mathrm{Myr}$ of the pre-main sequence evolution (e.g. D’Antona \& Mazzitelli 1997; Baraffe et al. 1998). In particular, the Li I $\lambda 670.8 \mathrm{~nm}$ absorption line is a well-known age indicator in young low-mass objects. This was shown, e.g., in studies of the line equivalent width for clusters and associations with different HR diagram ages (e.g. Mentuch et al. 2008). The age at which lithium depletes increases with decreasing mass. For the fully convective very lowmass $\left(<0.35 M_{\odot}\right)$ objects in star forming regions standard evolutionary models predict that the original abundances are still retained until an age of at least $10 \mathrm{Myr}$ (e.g. Jeffries 2006) and a cosmic abundance of $\mathrm{A}(\mathrm{Li}) \approx 3.1$ (Zapatero Osorio et al. 2002) is expected.

The LiI $\lambda 670.8 \mathrm{~nm}$ absorption of FU Tau A is shown in the top panel of Fig. 4. We determined an equivalent width of $0.43 \pm 0.02 \AA$. In practice, for accreting objects photospheric absorption lines may be filled in by excess continuum emission leading to shallower lines and an underestimate of the line equivalent widths. The amount of this "veiling" can be estimated by comparison with the spectrum of an unveiled star of the same spectral type. The only available template for FU Tau A observed with the same instrument and the same spectral resolution is Par-Lup3-1 (M6.5). We have thus measured the EWs of the TiO absorption bands in the region between $660 \mathrm{~nm}$ and $680 \mathrm{~nm}$ (Valenti et al. 1998) for both the template and FU Tau A. We calculated the ratio $E W_{\text {template }} / E W_{\text {FUTauA }}$ for eight different bands and found that the ratio is at most 1.2. A more detailed evaluation

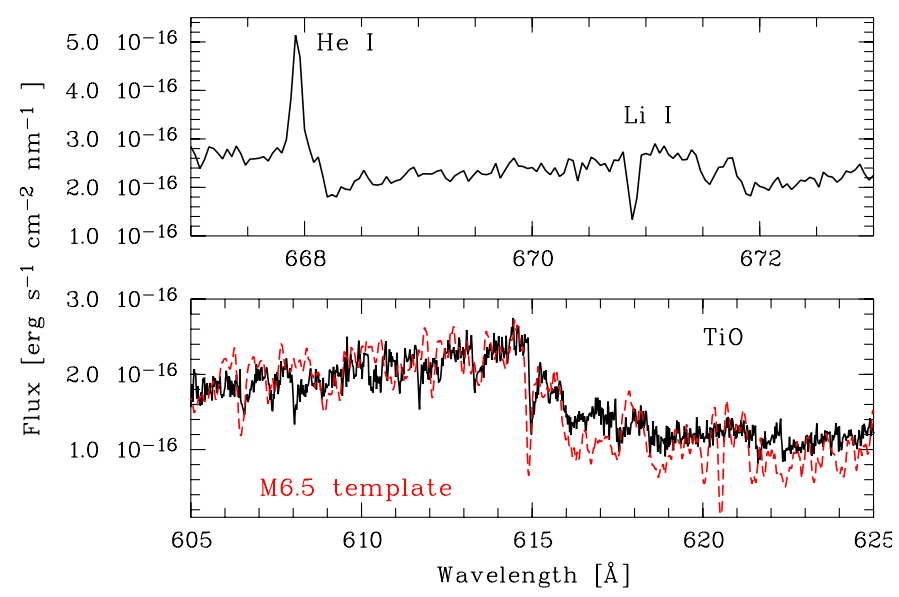

Fig. 4. Li I $\lambda 670.8 \mathrm{~nm}$ absorption in FU Tau A (top) and TiO absorption band at $615 \mathrm{~nm}$, a diagnostic of veiling, compared to the same spectral region in the unveiled Class III template Par-Lup3-1 (bottom).

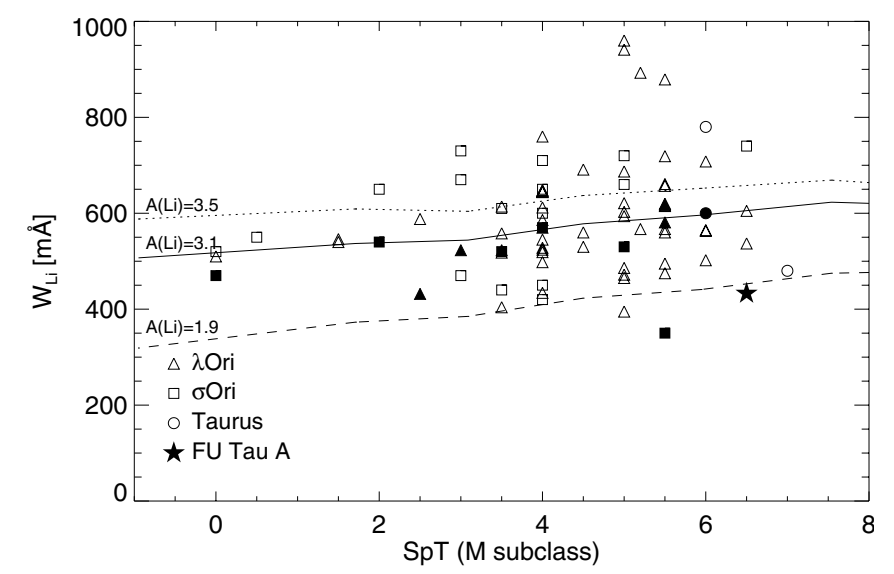

Fig. 5. Lithium equivalent width versus spectral type for FU Tau A (filled star symbol) and other measurements of M-type YSOs collected from the literature (see text in Sect. 3.3). Accretors are characterized by filled symbols. Also plotted are the curves of growth for $\log g=4.0$ and three different abundances with labels, calculated by Zapatero Osorio et al. (2002).

is prohibited by the noise level of the template spectrum. If these differences in EWs are due to veiling the corrected $E W_{\mathrm{Li}}$ value for FU Tau A would be $\lesssim 516 \mathrm{~m} \AA$. We have repeated the same exercise using the spectral features shown in the bottom panel of Fig. 4. The result is a correction factor of $\leq 1.3$ which is consistent with weak veiling.

In Fig. 5 we compare our measurement to other studies of lithium in YSOs of late-M spectral type from the literature: $\sigma$ Ori (Zapatero Osorio et al. 2002), $\lambda$ Ori (Bayo et al. 2011), and three very low-mass (VLM) objects in Taurus (Barrado y Navascués 2004). A compilation of lithium equivalent width measurements in Taurus is given by Sestito et al. (2008) for a larger range of spectral types. We do not consider this sample here because it includes a number of stars with dubious membership. For the case of $\lambda$ Ori we consider only the subsample with confirmed cluster membership and with spectroscopically determined spectral types.

Accreting objects are highlighted with filled plotting symbols in Fig. 5 and have been selected as follows: in $\sigma$ Ori we consider accreting all objects with $W_{\mathrm{H} \alpha}>20 \AA$, in $\lambda$ Ori we rely on the "accretor flag" given by Bayo et al. (2011) which is based on $\mathrm{H} \alpha$ emission and in Taurus we consider accreting 
Table 1. Proper motion of FU Tau A from the literature and Galactic velocity calculated using our RV measurement and $d=140 \mathrm{pc}$.

\begin{tabular}{lcc}
\hline \hline Reference & $\mu_{\alpha}, \mu_{\delta}$ & $(U, V, W)$ \\
& {$[\mathrm{mas} / \mathrm{yr}]$} & {$\left[\mathrm{km} \mathrm{s}^{-1}\right]$} \\
\hline Ducourant et al. (2005) & $14.7,-26.0$ & $(-22.09,-9.08,-10.36)$ \\
Luhman et al. (2009a) & $7.2,-17.5$ & $(-23.54,-16.55,-10.41)$ \\
\hline
\end{tabular}

objects with $W_{\mathrm{H} \alpha 10 \%}>300 \mathrm{~km} \mathrm{~s}^{-1}$. The choice of the $10 \%$ width rather than the equivalent width as accretion diagnostic for Taurus is motivated by the fact that one object, KPNO-Tau 5, has $W_{\mathrm{H} \alpha}=21.1 \AA$, at the borderline to the accretion regime but is classified as non-accretor due to the narrow $\mathrm{H} \alpha$ profile and weak He I $\lambda 667.8$ emission.

The above-mentioned star forming regions all have ages of 1-5 Myr, and the lithium content of M stars is expected to reflect the initial cosmic abundance, $\mathrm{A}(\mathrm{Li})=3.1$. However, a wide spread of equivalent widths is observed for a given spectral type. Observations obtained with different spectral resolutions may result in systematic differences in the line measurements, but these errors are smaller than the observed spread of the equivalent width values. This spread also corresponds to an unexplained spread in abundances as is evident by the overplotted curves of growth for $\log g=4.0$ calculated by Zapatero Osorio et al. (2002). FU Tau A presents the lowest Li measurement observed so far for its spectral class. When taking veiling into account, FU Tau A moves up in the diagram but is still well below the theoretically expected abundance.

\subsection{Radial velocity (RV) and UVW velocity}

We used the strongest absorption lines, the KI doublet at $\lambda \lambda$ 766.48, $769.89 \mathrm{~nm}$ and the $\mathrm{Na}$ I doublet at $\lambda \lambda$ 818.33, $819.48 \mathrm{~nm}$ to estimate the radial velocity of FU Tau A by comparing the observed line centers to the rest wavelengths extracted from the NIST Atomic Spectra Database ${ }^{3}$. After application of the barycentric correction $\left(-20.6 \mathrm{~km} \mathrm{~s}^{-1}\right)$, the mean Doppler shift of these lines yields $R V=22.5 \pm 2.9 \mathrm{~km} \mathrm{~s}^{-1}$ where we have added to the standard deviation of the four measured lines the uncertainty of the wavelength calibration (see Sect. 2). The local-standard-of-rest velocity of FU Tau A is $12.5 \pm 2.9 \mathrm{~km} \mathrm{~s}^{-1}$.

Bertout \& Genova (2006) have compiled a list of 127 stars in the Taurus star forming complex with RV measurements. These authors note that no systematic high-precision RV survey has been performed in Taurus. Their RV distribution is strongly peaked at $15-16 \mathrm{~km} \mathrm{~s}^{-1}$, in agreement with historic smaller samples presented by Hartmann et al. (1986) and Walter et al. (1988). About $\sim 5 \%$ of this list have $R V \geq 22 \mathrm{~km} \mathrm{~s}^{-1}$; i.e., the RV of FU Tau A is only marginally compatible with that of Taurus.

We combined our RV measurement with the proper motion of FU Tau A given in the literature to obtain the $U V W$ velocities. We used both the proper motion values of Ducourant et al. (2005) and those of Luhman et al. (2009a). The resulting galactic velocity components for a distance of $140 \mathrm{pc}$ are listed in Table 1. The average space motion for Taurus for the same distance is $(U, V, W)=(-16.5 \pm 4.6,-13.2 \pm 2.5,-11.0 \pm 4.0) \mathrm{km} \mathrm{s}^{-1}$ (Bertout \& Genova 2006). FU Tau A roughly agrees with these values.

\footnotetext{
3 http://www.nist.gov/pml/data/asd.cfm/
}

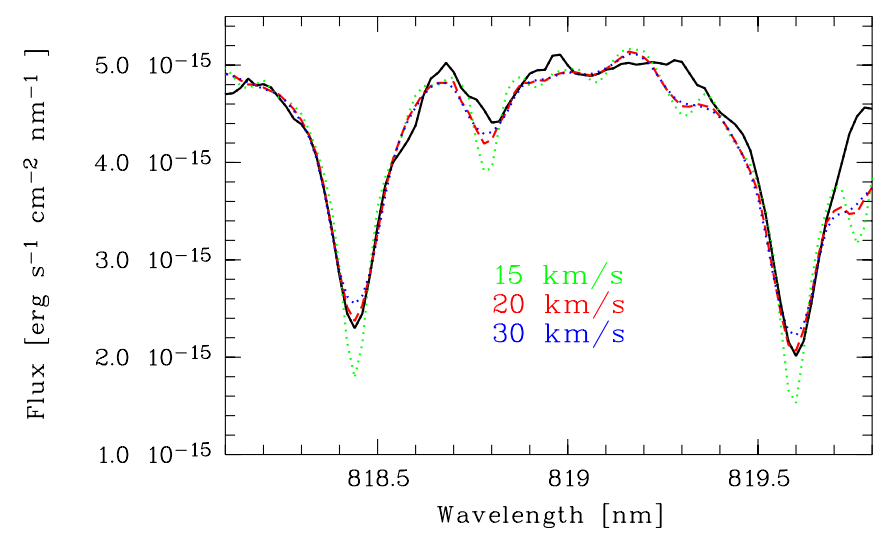

Fig. 6. The Na I absorption doublet $\lambda \lambda 818.33,819.48 \mathrm{~nm}$ of FU Tau A is shown as a continuous black line. The synthetic spectrum, broadened at projected rotational velocities of 15,20 , and $30 \mathrm{~km} \mathrm{~s}^{-1}$, is represented with the green-dotted, red-dashed, and blue-dotted lines, respectively.

\subsection{Rotational velocity}

The projected rotational velocity, $v \sin i$, was estimated by comparing the profile of the $\mathrm{NaI}$ absorption doublet at $\lambda \lambda 818.33,819.48 \mathrm{~nm}$ with that of a synthetic BT-DUSTY spectrum of the same effective temperature and gravity as FU Tau A. The synthetic spectrum was gathered from the star, brown dwarf $\mathcal{E}$ planet atmosphere web simulator (Allard et al. 2012) using a $v \sin i=0 \mathrm{~km} \mathrm{~s}^{-1}$. To reproduce the profile of the $\mathrm{Na}$ I absorption doublet in FU Tau A, the synthetic spectrum was convolved with rotational profiles (see Gray 1992) of several values of $v \sin i$. The best match is for $v \sin i=20 \mathrm{~km} \mathrm{~s}^{-1}$ (see Fig. 6). We estimate an error on the order of $5 \mathrm{~km} \mathrm{~s}^{-1}$.

We can combine our measurements of the rotational velocity $(v \sin i)$ with the photometrically determined rotation period from Scholz et al. (2012) $(P=4.0 \pm 0.2 \mathrm{~d})$ and find $R \sin i=$ $1.6 \pm 0.5 R_{\odot}$. The value obtained for $R \sin i$ is compatible with our new value for the stellar radius $\left(R_{*}=1.4 R_{\odot}\right)$ within the errors and implies a disk inclination angle $i \geq 53^{\circ}$.

\section{Outflows}

We searched for forbidden emission lines (FELs) as signatures of shocks forming in outflows. A list of the equivalent widths and line fluxes for the FELs detected in FU Tau A is given in Cols. 3 and 4 of Table 2. The EWs and fluxes are obtained from a by-eye estimate of the local continuum. The uncertainties of the EWs and the fluxes represent the mean and standard deviations from three measurements carried out on each line. In our approach of estimating these uncertainties, we account for the uncertainties in the flux being dominated by the uncertainty of the extinction, while the major source of the uncertainties in the EWs are the statistical fluctuations. Therefore, for the errors of the EWs, we measured the line in the spectrum dereddened by $0.5 \mathrm{mag}$ at another two positions corresponding to our estimate of the upper and lower values of the adjacent continuum emission and computed the standard deviation of the three measurements. For the uncertainties of the line fluxes we measured the line in the observed spectrum (corresponding to an assumption of $A_{\mathrm{V}}=0 \mathrm{mag}$ ) and in the spectrum dereddened for $A_{\mathrm{V}}=1 \mathrm{mag}$, and combined those two values with that from the spectrum dereddened by $0.5 \mathrm{mag}$ to get the standard deviation.

Not detecting the [SII] $\lambda 671.6 \mathrm{~nm}$ line does not allow us to apply the technique of Bacciotti \& Eislöffel (1999) for estimating plasma parameters from the line ratios of FELs. For a 
Table 2. Forbidden emission lines.

\begin{tabular}{lcccccc}
\hline \hline Element & $\begin{array}{c}\lambda_{0} \\
{[\mathrm{~nm}]}\end{array}$ & $\begin{array}{c}E W \\
{[\mathrm{~nm}]}\end{array}$ & $\begin{array}{c}f_{\text {line }} \cdot 10^{16} \\
{\left[\mathrm{erg} / \mathrm{cm}^{2} / \mathrm{s}\right]}\end{array}$ & $\begin{array}{c}R V \\
{\left[\mathrm{~km} \mathrm{~s}^{-1}\right]}\end{array}$ & $\begin{array}{c}V_{\mathrm{T}} \\
{\left[\mathrm{km} \mathrm{s}^{-1}\right]}\end{array}$ & $\begin{array}{c}\Delta V \\
{\left[\mathrm{~km} \mathrm{~s}^{-1}\right]}\end{array}$ \\
\hline SII & 406.860 & $-0.305 \pm 0.102$ & $2.7 \pm 1.5$ & -26.6 & 99.2 & 3.2 \\
SII & 407.635 & $-0.081 \pm 0.083$ & $0.4 \pm 0.2$ & $\ldots$ & $\ldots$ & $\ldots$ \\
OI & 630.030 & $-0.323 \pm 0.053$ & $8.8 \pm 3.4$ & -12.6 & 47.2 & 3.2 \\
OI & 636.378 & $-0.067 \pm 0.011$ & $2.7 \pm 1.0$ & $\ldots$ & $\ldots$ & $\ldots$ \\
NII & 658.260 & $-0.009 \pm 0.007$ & $0.7 \pm 0.4$ & $\ldots$ & $\ldots$ & $\ldots$ \\
SII & 673.082 & $-0.030 \pm 0.010$ & $1.2 \pm 0.4$ & -11.0 & 41.1 & 3.2 \\
\hline
\end{tabular}

Notes. Nominal wavelength (Col. 2), line equivalent width and flux (Cols. 3 and 4), radial velocity with respect to the barycenter-corrected stellar velocity (Col. 5), tangential velocity (Col. 6) and uncertainty of the velocities.

detailed analysis of luminosities, kinematics, and mass outflow rate $\left(\dot{M}_{\text {out }}\right)$ of FU Tau A, we consider only the two most prominent lines, [OI] $\lambda 630.0 \mathrm{~nm}$ and [SII] $\lambda 673.1 \mathrm{~nm}$, and we follow the prescription of Hartigan et al. (1995) who presented relations of the type

$\log \dot{M}_{\text {out }}=X \cdot\left(1+\frac{n_{\mathrm{c}}}{n_{\mathrm{e}}}\right)\left(\frac{V_{\mathrm{T}}}{150 \mathrm{kms}^{-1}}\right)\left(\frac{l_{\mathrm{T}}}{2 \times 10^{15} \mathrm{~cm}}\right)^{-1}\left(\frac{L_{\text {line }}}{L_{\text {sun }}}\right)$.

The numerical constant $X$ was derived in Hartigan et al. (1995) for the $[\mathrm{SII}] \lambda 673.1 \mathrm{~nm}$, and the [OI] $\lambda 630.0 \mathrm{~nm}$ lines. Equation (1) involves the electron density $\left(n_{\mathrm{e}}\right)$, the tangential velocity of the outflow $\left(V_{\mathrm{T}}\right)$, and the projected size of the aperture in the plane of the sky $\left(l_{\mathrm{T}}\right)$. For a $1^{\prime \prime}$ slit we obtain for the distance of Taurus $l_{\mathrm{T}}=2 \times 10^{15} \mathrm{~cm}$. For the reasonable assumption that the outflow is perpendicular to the disk we use the minimum possible disk inclination angle $\left(i_{\text {disk }} \sim 50^{\circ}\right.$; see Sect. 3.5$)$ and the measured RV of the FELs with respect to the stellar RV to estimate the tangential velocities. The derived quantities are given in Table 2. The uncertainty in the velocities are given in Col. 7. They include the standard deviation of three wavelength measurements and the uncertainty of the stellar RV.

We have no measurement of the electron density for the outflow of FU Tau A. A lower limit to $n_{\mathrm{e}}$ is given by the fact that we detect [SII] $\lambda 673.1 \mathrm{~nm}$ but not [SII] $\lambda 617.7 \mathrm{~nm}$ emission. The flux ratio between these two lines is, therefore, greater than unity and $n_{\mathrm{e}}>10^{3} \mathrm{~cm}^{-3}$ (e.g. Osterbrock 1989). This gives an upper limit to the mass loss rate determined from [SII] $\lambda 673.1 \mathrm{~nm}$ of $\log \dot{M}_{\text {out }}\left[M_{\odot} /\right.$ yr $]<-10.4$. Assuming the same lower limit for the electron density, the [OI] $\lambda 630.0 \mathrm{~nm}$ line gives a much less stringent constraint on $\dot{M}_{\text {out }}$. We note that the mass loss rates computed from the two lines agree for $n_{\mathrm{e}} \sim 10^{5} \mathrm{~cm}^{-3}$. For that case, we find $\log \dot{M}_{\text {out }}\left[M_{\odot} / \mathrm{yr}\right]<-11.5$. Recall also that the value of the inclination angle assumed in this calculation is the minimum possible value on the basis of the rotational properties of FU Tau A. If the disk inclination is higher than that value, the upper limit on the mass loss rate is higher and less meaningful; e.g., for $i=75^{\circ}$ the mass loss rate obtained from [SII] is $<10^{-10.0} M_{\odot} /$ yr for $n_{\mathrm{e}}>10^{3} \mathrm{~cm}^{-3}$ and $<10^{-11.0} M_{\odot} / \mathrm{yr}$ for $n_{\mathrm{e}} \sim 10^{5} \mathrm{~cm}^{-3}$.

\section{Accretion}

Different methods have been described for estimating the mass accretion rate $\left(\dot{M}_{\text {acc }}\right)$ from the properties of emission lines or continuum emission. The various accretion diagnostics probe several physical regions; e.g., the excess continuum that veils the absorption features of classical $\mathrm{T}$ Tauri stars is ascribed to accretion shocks (Calvet \& Gullbring 1998) and the highexcitation HeI emission to post-shock regions (Beristain et al. 2001). Hydrogen lines from the Paschen and Balmer series are produced in accretion flows, with $\mathrm{H} \alpha$ having the largest optical depth, originating in their outermost parts (Hartmann et al. 1994; Muzerolle et al. 1998).

Here, we use two approaches for measuring the mass accretion rate of FU Tau A. We make use of empirical relations with emission line fluxes and luminosities (Sect. 5.2.1) and model the continuum excess (Sect. 5.2.2).

\subsection{Emission line analysis}

Using IRAF we measured equivalent widths and line fluxes of the dominant emission lines in the spectrum in the same manner as described in Sect. 4 for the case of the FELs. The by far strongest line in the X-shooter spectrum of FU Tau A is $\mathrm{H} \alpha$. We measure an equivalent width of $93 \pm 5 \AA$. Previous $\mathrm{H} \alpha$ measurements of FU Tau A reported in the literature, all based on lower resolution spectra, range between 93 and $155 \AA$ (Luhman et al. 2009b; Stelzer et al. 2010; Scholz et al. 2012). Considering that low-resolution observations tend to underestimate line equivalent widths, this points at only modest variability. The Balmer series can be reliably identified up to $n=16$. No lines of the Paschen or Brackett series are detected.

Some of the Balmer line profiles are displayed in Fig. 7. All Balmer lines including $\mathrm{H} \alpha$ are single-peaked. The lines in Fig. 7 represent the expected line center corrected for barycenter motion and the systemic RV of FU Tau A derived in Sect. 3.4. While the $\mathrm{H} \alpha$ profile is nearly symmetric, the other low-n Balmer lines show small asymmetries with a flux deficiency on the red side of the profile. This might be interpreted as red absorption, a typical signature of infall (Hartmann et al. 1994).

\subsection{Mass accretion rates}

\subsubsection{Emission line diagnostics}

The published calibrations between emission line strength and mass accretion rate come in two flavors. The first consists of relations between line luminosity $\left(L_{\text {line }}\right)$ and accretion luminosity $\left(L_{\mathrm{acc}}\right)$ of the type

$\log \left(L_{\text {acc }} / L_{\odot}\right)=a \cdot \log \left(L_{\text {line }} / L_{\odot}\right)+b$.

This can be converted to an accretion rate according to

$\dot{M}_{\mathrm{acc}}=1.25 \cdot \frac{L_{\mathrm{acc}} \cdot R_{*}}{G \cdot M_{*}}$, 

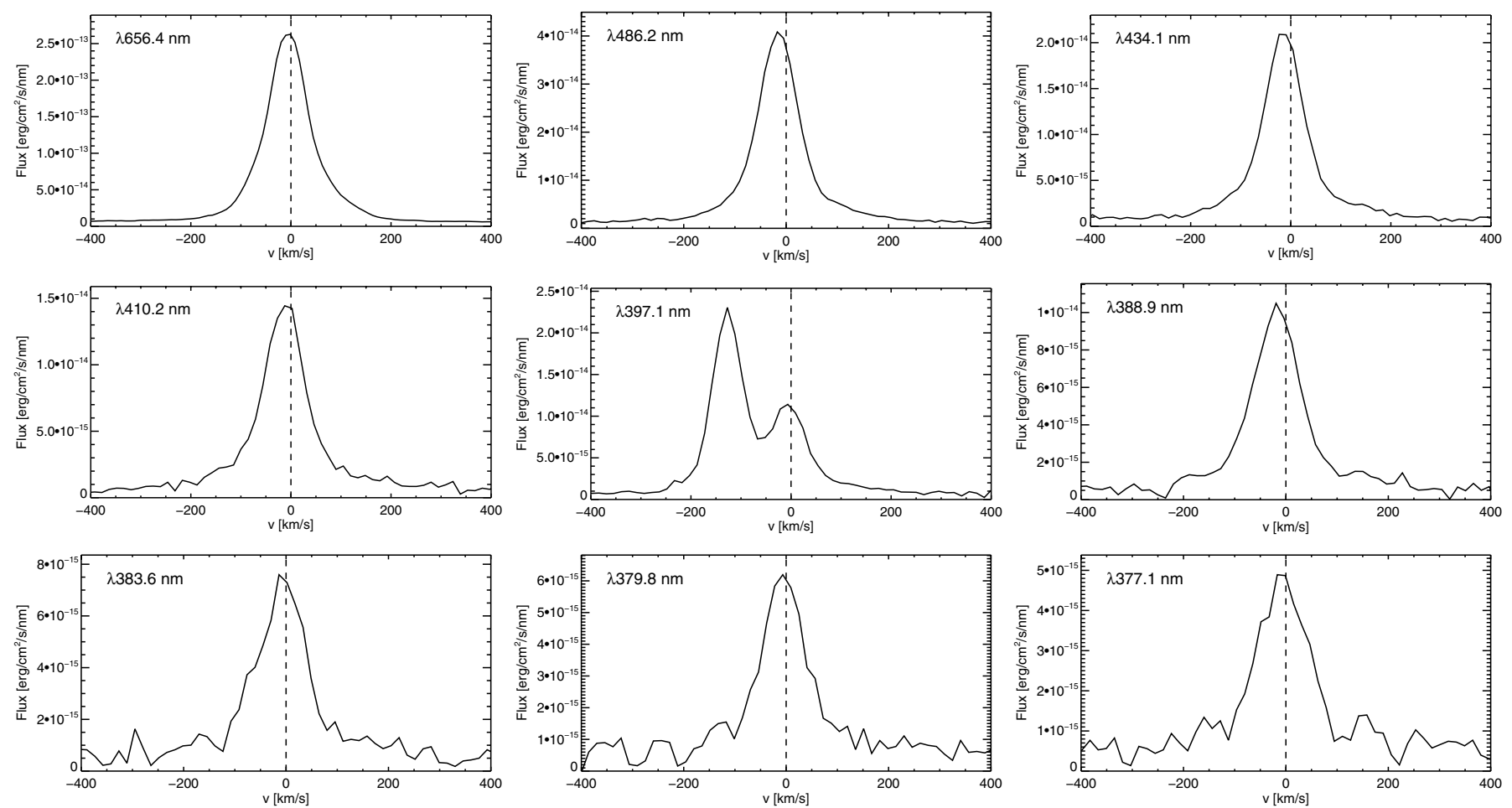

Fig. 7. Line profiles for the lower Balmer series of FU Tau A. The dashed vertical line represents the expected line center correcting for the barycenter motion and the stellar RV computed from absorption lines.

where the numerical factor derives from the assumption for the inner disk radius, $R_{\text {in }}=5 R_{*}$ (Gullbring et al. 1998). The second approach relates the line surface flux $\left(F_{\mathrm{s}, \text { line }}\right)$ directly to the accretion rate in the form of

$\log \dot{M}_{\mathrm{acc}}=c \cdot \log F_{\mathrm{s}, \text { line }}+d$.

The coefficients $a, b, c$, and $d$ have been derived for an increasing sample of YSOs, however, so far without consensus between the various studies.

To estimate $\dot{M}_{\text {acc }}$ for FU Tau A, we used the published relations presented by Herczeg \& Hillenbrand (2008), Mohanty et al. (2005), Rigliaco et al. (2012, hereafter RNT12) and new calibrations by Alcalá et al. (in prep.). The last are based on an X-shooter sample of 36 accreting YSOs from the Lupus star forming region, and they represent the largest homogeneous analysis of this kind performed so far. The distance used to convert line fluxes to luminosities is that of the Taurus star forming complex, $140 \mathrm{pc}$. The mass and radius of FU Tau A required for evaluating Eqs. (3) and (4) are given in Sect. 3.2. The derived mass accretion rates for all detected emission lines in common with the relations presented in the literature are shown in Fig. 8. Each literature source is marked with a different plotting symbol. Some values that deviate strongly from the bulk of the measurements are not shown for clarity but are discussed below. The error bars again represent the uncertainty in the reddening with upper and lower bounds corresponding to $A_{\mathrm{V}}=0$ and $A_{\mathrm{V}}=1 \mathrm{mag}$, respectively.

We calculated the mass accretion rate for all emission lines detected in the X-shooter spectrum of FU Tau A that have been calibrated in the literature. However, we computed the average of $\dot{M}_{\text {acc }}$ only from those lines that we consider the most reliable accretion diagnostics. In particular, we excluded the Ca II IRT lines and $\mathrm{NaI} \mathrm{D}$, for which strongly discrepant accretion rates are found with different calibrations. Some of the values for $\dot{M}_{\text {acc }}$ that we have derived for these lines are outside the range of values shown in Fig. 8. For a detailed investigation of the reliability of individual emission lines as an accretion diagnostic see, e.g., Alcalá et al. (in prep.). We also did not take [O I] $\lambda 630 \mathrm{~nm}$ and $\mathrm{H} \alpha$ into account, which are often affected by winds in YSOs (see Sect. 4). Ca II $\mathrm{H}$ and $\mathrm{H} \epsilon$ are also excluded because these lines are partially blended in our spectrum. The average obtained from the remaining lines is $\left\langle\log \dot{M}_{\text {acc }}\right\rangle\left[M_{\odot} / \mathrm{yr}\right]=-9.9 \pm 0.2$ for 20 lines from Alcalá et al., $\left\langle\log \dot{M}_{\mathrm{acc}}\right\rangle\left[M_{\odot} / \mathrm{yr}\right]=-10.2 \pm 0.2$ for four lines from RNT12, and $\left\langle\log \dot{M}_{\text {acc }}\right\rangle\left[M_{\odot} / \mathrm{yr}\right]=-10.2 \pm 0.2$ for six lines from HH08. Here the uncertainties represent the standard deviation of all measurements. The mean and standard deviation of the calibrations by Alcalá et al. are overplotted in Fig. 8. The influence of the uncertainties of mass and distance on this result is examined in Sect. 6. We anticipate here that they make $\log \dot{M}_{\text {acc }}$ change only marginally. Similarly, a lower value for the inner disk radius, e.g. $R_{\text {in }}=2 R_{\odot}$, would yield a mass accretion rate that is marginally compatible with the value given above.

We have also derived the mass accretion rate from the $\mathrm{H} \alpha$ $10 \%$ width. The measured full width half maximum at $10 \%$ of the peak height is $228 \mathrm{~km} \mathrm{~s}^{-1}$. For the calibration provided by Natta et al. (2004), this corresponds to a mass accretion rate of $\log \dot{M}_{\text {acc }}\left[M_{\odot} /\right.$ yr $]=-10.7 \pm 0.5$. This value, also shown in Fig. 8, is almost one order of magnitude lower than the mean value derived from the relations presented by Alcalá et al.

\subsubsection{Continuum excess}

The accretion luminosity can be estimated directly by measuring the continuum emission in excess of the photospheric one. This is done by comparing it to a nonaccreting template star of the same spectral type. Unfortunately, the Class III Par-Lup3-1 that we used as spectral template in Sect. 3.1 is too noisy to be used 


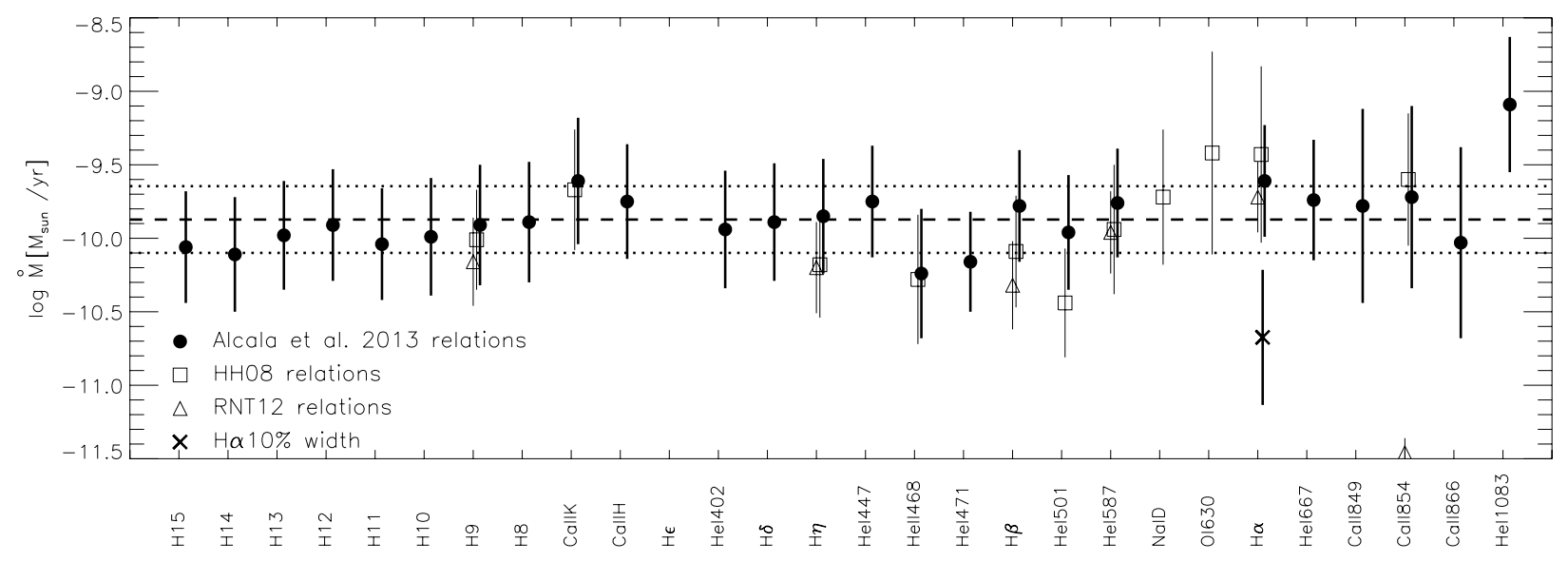

Fig. 8. Mass accretion rate for FU Tau A derived from various emission line fluxes and luminosities and from the $10 \%$ width of $\mathrm{H} \alpha$ using different calibrations provided in the literature as labeled in the figure. Dashed and dotted lines represent the mean and standard deviation for the relations from Alcalá (in prep.) using 20 emission lines as described in Sect. 5.2.1.

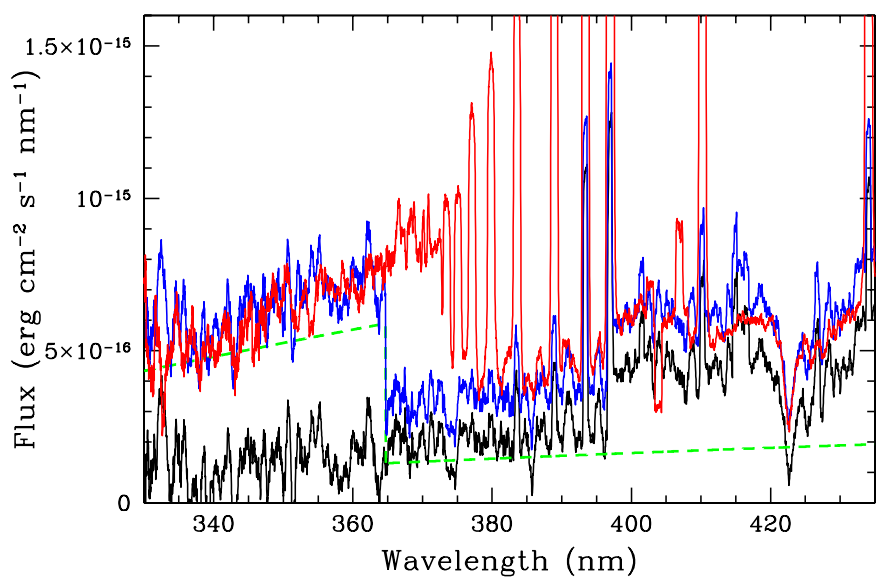

Fig. 9. Balmer jump region in the spectrum of FU Tau A, heavily smoothed to reduce the errors on the continuum (red), the Class III template SO 925 (black), the excess emission from the accretion slab model (dashed green) and the resulting modeled emission, i.e. template plus excess emission, in blue. All spectra are normalized in the region $700-720 \mathrm{~nm}$. The observed emission in the range $364.6 \mathrm{~nm}$ to $\sim 370 \mathrm{~nm}$ is dominated by unresolved Balmer lines, which are not included in the slab model.

for this purpose. Figure 9 shows the blue part of the spectrum of FU Tau A compared to that of another young Class III star of similar (albeit slightly earlier) spectral type, SO 925 (M5.5; see RNT12 and MTR13 normalized in the region 700-720 nm. FU Tau A shows a clear excess emission in the Balmer continuum and a relatively small Balmer jump, defined as the ratio of the fluxes observed in the continuum at $360 \mathrm{~nm}$ and at $420 \mathrm{~nm}$, of $\sim 1.2$. The Balmer limit is at $364.6 \mathrm{~nm}$, but owing to the blending of the higher Balmer lines it appears shifted to $370 \mathrm{~nm}$.

We model the excess emission as caused by a slab of hydrogen of given electron density, temperature, and length (see e.g., Valenti et al. 1993, HH08, RNT13) and obtain an estimate of the excess continuum luminosity of $L_{\text {acc }} \sim 1.3 \times 10^{-4} L_{\odot}$. Figure 9 shows the best-fitting model in blue. It reproduces quite well the observed Balmer continuum, the Balmer jump, and the profile of the Ca II line at $420 \mathrm{~nm}$. Veiling of photospheric lines at longer wavelengths is very weak as shown in Sect. 3.3. The uncertainties of $L_{\text {acc }}$ are quite large (50\% at least), due to the noise of the spectra of both FU Tau A and the Class III template and to the mismatch between the spectral type of FU Tau A and that of the template. For $M_{*}=0.08 M_{\odot}$ and $R_{*}=1.38 R_{\odot}$ as determined in Sect. 3.2, the corresponding mass accretion rate from Eq. (3) is $\log \dot{M}_{\text {acc }}\left[M_{\odot} /\right.$ yr $] \sim-10.1$, in reasonably good agreement with the estimates from the emission lines presented in Sect. 5.2.1.

\section{Discussion}

We have analyzed a broad-band (300-2480 nm) mediumresolution $(R \sim 5000-9000)$ spectrum of FU Tau A obtained with $\mathrm{X}$-shooter. On the basis of these data, we have presented the first measurements of gravity, radial velocity, rotational velocity, and lithium content, as well as the first detection of outflow activity in FU Tau A. Moreover, this spectrum provides a large number of accretion diagnostics that we have employed for a detailed evaluation of the mass accretion rate.

The motivation for our spectroscopic study was to search for an explanation of the (apparent) overluminosity of FU Tau A with respect to the predictions of evolutionary models for its age and mass. This requires precise measurements of the fundamental stellar parameters $\left(T_{\mathrm{eff}}, L_{\mathrm{bol}}, R_{*}, \log g\right)$, as well as a study of other phenomena, such as accretion and magnetic activity that may lead to a wrong estimate of those parameters. We have discussed several possible factors that may influence the position of FU Tau A in the HR diagram in our previous papers (Stelzer et al. 2010; Scholz et al. 2012), and we resume this discussion here, adding the wealth of information provided by the $\mathrm{X}$-shooter spectrum.

\subsection{Accretion and outflow in FU Tau A}

In our detailed investigation of mass accretion in FU Tau A, we made use of an unprecedentedly large number of empirical relations between the luminosity of individual emission lines and the accretion luminosity derived from a comprehensive X-shooter study of accretors in the Lupus clouds (Alcalá et al., in prep.). These relations comprise emission features collected with all three arms of the X-shooter spectrograph. As compared to similar relations presented in the literature, we consider them the most reliable calibrations for our observation of FU Tau A given that they were derived with the same instrument. A mean value of $\left\langle\log \dot{M}_{\text {acc }}\right\rangle\left[M_{\odot} / \mathrm{yr}\right]=-9.9 \pm 0.2$ is found from a total of 20 emission lines. The accretion rate obtained from the $\mathrm{H} \alpha 10 \%$ width $\left(\log \dot{M}_{\text {acc, } 10 \%}\left[M_{\odot} / \mathrm{yr}\right]=-10.7 \pm 0.5\right)$ is lower by almost one order of magnitude. The discrepancy of $\log \dot{M}_{\text {acc, } 10 \%}$ with 
Table 3. Equivalent widths and fluxes for emission lines.

\begin{tabular}{lrcc}
\hline \hline Line & $\begin{array}{r}\lambda_{0} \\
{[\mathrm{~nm}]}\end{array}$ & $\begin{array}{c}E W \\
{[\mathrm{~nm}]}\end{array}$ & $\begin{array}{c}\log f_{\text {line }} \\
{\left[\mathrm{erg} / \mathrm{cm}^{2} / \mathrm{s}\right]}\end{array}$ \\
\hline HeI 1083 & 1083.038 & $-0.150 \pm 0.026$ & $-13.73_{-0.07}^{+0.06}$ \\
CaII 866 & 866.381 & $-0.017 \pm 0.003$ & $-15.11_{-0.11}^{+0.10}$ \\
CaII 854 & 854.347 & $-0.076 \pm 0.015$ & $-14.61_{-0.12}^{+0.10}$ \\
CaII 849 & 849.936 & $-0.042 \pm 0.010$ & $-14.86_{-0.12}^{+0.10}$ \\
HeI 667 & 667.928 & $-0.089 \pm 0.014$ & $-15.40_{-0.14}^{+0.18}$ \\
H $\alpha$ & 656.374 & $-9.766 \pm 0.709$ & $-13.18_{-0.16}^{+0.17}$ \\
[OI]630 & 630.093 & $-0.314 \pm 0.039$ & $-15.09_{-0.19}^{+0.15}$ \\
NaI D1 & 589.697 & $-0.221 \pm 0.044$ & $-15.51_{-0.21}^{+0.18}$ \\
NaI D2 & 589.096 & $-0.372 \pm 0.067$ & $-15.27_{-0.20}^{+0.18}$ \\
HeI 587 & 587.670 & $-0.581 \pm 0.065$ & $-15.01_{-0.18}^{+0.20}$ \\
HeI 501 & 501.625 & $-0.071 \pm 0.016$ & $-16.07_{-0.24}^{+0.18}$ \\
H $\beta$ & 486.184 & $-6.360 \pm 1.765$ & $-14.14_{-0.23}^{+0.22}$ \\
HeI 471 & 471.397 & $-0.031 \pm 0.009$ & $-16.50_{-0.24}^{+0.15}$ \\
HeII 468 & 468.633 & $-0.048 \pm 0.016$ & $-16.30_{-0.23}^{+0.20}$ \\
HeI 447 & 447.216 & $-0.470 \pm 0.164$ & $-15.47_{-0.23}^{+0.24}$ \\
H $\gamma$ & 434.096 & $-6.334 \pm 2.929$ & $-14.44_{-0.23}^{+0.24}$ \\
H $\delta$ & 410.221 & $-4.151 \pm 2.327$ & $-14.64_{-0.26}^{+0.22}$ \\
HeI 402 & 402.687 & $-0.238 \pm 0.120$ & $-15.87_{-0.26}^{+0.25}$ \\
H $\epsilon$ & 397.068 & $-1.767 \pm 1.078$ & $-14.89_{-0.26}^{+0.25}$ \\
CaII H & 396.898 & $-5.297 \pm 3.764$ & $-14.63_{-0.20}^{+0.28}$ \\
CaII K & 393.419 & $-5.981 \pm 2.717$ & $-14.52_{-0.25}^{+0.25}$ \\
H8 & 388.944 & $-4.875 \pm 3.823$ & $-14.78_{-0.25}^{+0.25}$ \\
H9 & 383.588 & $-2.392 \pm 1.852$ & $-14.98_{-0.24}^{+0.26}$ \\
H10 & 379.845 & $-0.975 \pm 0.447$ & $-15.15_{-0.26}^{+0.24}$ \\
H11 & 377.113 & $-0.855 \pm 0.512$ & $-15.25_{-0.27}^{+0.22}$ \\
H12 & 375.071 & $-1.230 \pm 0.684$ & $-15.28_{-0.26}^{+0.24}$ \\
H13 & 373.484 & $-0.658 \pm 0.422$ & $-15.44_{-0.25}^{+0.18}$ \\
H14 & 372.242 & $-0.384 \pm 0.250$ & $-15.66_{-0.27}^{+0.19}$ \\
H15 & 371.232 & $-0.377 \pm 0.276$ & $-15.64_{-0.28}^{+0.25}$ \\
\hline & 370.432 & $-0.105 \pm 0.057$ & $-16.04_{-0.25}^{+0.22}$ \\
\hline & & &
\end{tabular}

respect to other accretion diagnostics supports previous notions of the poor reliability of this tracer (e.g., Costigan et al. 2012). On the other hand, the accretion rate from the $\mathrm{H} \alpha 10 \%$ width as measured in the $\mathrm{X}$-shooter spectrum is also much lower than the value measured with the same diagnostics from a previous Gemini spectrum $\left(\log \dot{M}_{\mathrm{acc}, 10 \%}\left[M_{\odot} / \mathrm{yr}\right]=-9.5\right.$; Stelzer et al. 2010). The low velocities in the new $\mathrm{H} \alpha$ profile are not sufficient to explain the temperature of the X-ray emitting plasma observed by Stelzer et al. (2010) as originating in an accretion shock.

We have presented the first evidence of outflow activity in FU Tau A in the form of several forbidden emission lines. The outflow of FU Tau A has recently also been detected at millimeter wavelengths (Monin et al. 2013). In this paper, we have used the [OI] $\lambda 630.0 \mathrm{~nm}$ and [SII] $\lambda 673.1 \mathrm{~nm}$ lines detected in the $\mathrm{X}$-shooter spectrum to derive the mass loss rate of the outflow using the approach presented by Hartigan et al. (1995). We trust the result from [SII] $\lambda 673.1 \mathrm{~nm}$ more than that from $[\mathrm{OI}] \lambda 630.0 \mathrm{~nm}$ because the former has a weaker dependence on the unknown electron density. An additional uncertainty is introduced by the fact that we only have a lower limit on the disk inclination angle that determines the transverse outflow velocity. Assuming $i=53^{\circ}$ and $n_{\mathrm{e}} \sim 10^{-3} \mathrm{~cm}^{-2}$, we derive a mass outflow rate of $\log \dot{M}_{\text {out }}\left[M_{\odot} / \mathrm{yr}\right] \sim-10.4$ from the [SII] $\lambda 673.1 \mathrm{~nm}$ flux. Combining this with the contemporaneous measurement of the mass accretion rate, we find an outflow-toinflow rate $\dot{M}_{\text {out }} / \dot{M}_{\text {acc }} \sim 0.3$. This value for the mass outflow rate of FU Tau A could be both a lower limit (given that $n_{\mathrm{e}}$ is an upper limit) and an upper limit (given that $i$ is a lower limit); see Sect. 4 for details. Very few measurements of $\dot{M}_{\text {out }} / \dot{M}_{\text {acc }}$ have been presented in the literature for VLM objects. While some of these observations suggest that mass inflow and outflow rate are similar in the VLM regime (Bacciotti et al. 2011; Whelan et al. 2009), others point at results close to the "canonical" value for higher mass classical T Tauri stars of $\dot{M}_{\text {out }} / \dot{M}_{\text {acc }} \sim 0.1 \ldots 0.01$ (Joergens et al. 2012). A final conclusion on the comparison of the mass outflow to inflow ratio between BDs and cTTSs and the comparison of observations to the predictions of jet-launching models (Cabrit 2009) is premature considering the large uncertainties associated with the observations of $\dot{M}_{\text {out }}$ and $\dot{M}_{\text {acc }}$ in VLM objects and the inhomogeneous approach by different authors for calculating these numbers.

Signs for in- and outflows of FU Tau A are also evident from the line profiles and line shifts observed in the X-shooter spectrum. The modest deficiency of flux on the red side of the low-n Balmer lines is in qualitative agreement with the outcome of magnetospheric accretion models for high inclination. The calculations by Hartmann et al. (1994) predict a lack of flux in the red wing of optically thin emission lines that increases with decreasing inclination angle due to occultation of the receeding part of the accretion flow by the disk. The strongest forbidden lines show a similar blueshift of $\approx-10 \mathrm{~km} \mathrm{~s}^{-1}$ with respect to the stellar motion. This emission likely represents the approaching lobe of the jet, while the receeding (redshifted) part of the outflow may be occulted by the accretion disk. These are plausible interpretations that are consistent with our range for the disk inclination angle (derived from $v \sin i$, rotation period, and stellar radius) that excludes a pole-on view.

\subsection{FU Tau A in the HR diagram}

\subsubsection{Extinction}

A wrong extinction estimate could, in principle, misplace the object in the HR diagram in the vertical direction. We determined the spectral type of FU Tau A to M6.5 \pm 0.4 and the optical extinction to $A_{\mathrm{V}}=0.5 \pm 0.5 \mathrm{mag}$. This is slightly hotter and less absorbed than found by Luhman et al. (2009a), and results in a higher effective temperature $\left(T_{\text {eff }}=2940 \mathrm{~K}\right)$ and lower bolometric luminosity $\left(L_{\text {bol }}=0.13 L_{\odot}\right)$ and radius $\left(R_{*}=1.38 R_{\odot}\right)$. The lower extinction moves the object vertically down in the HR diagram by less than a factor two. This is hardly sufficient for resolving the luminosity problem of FU Tau A.

\subsubsection{Accretion}

A possible explanation for the overluminosity of FU Tau A with respect to the youngest isochrones of evolutionary models could be an overestimate of the bolometric luminosity due to a substantial contribution of accretion luminosity. In Scholz et al. (2012) we argued that this scenario is unlikely because of the relatively low temperature derived for the accretion hot spots. It was also 
pointed out that a significantly lower value for $L_{\mathrm{bol}}$, when combined with the X-ray data presented by Stelzer et al. (2010), would yield an exceptionally high X-ray to bolometric flux ratio. The X-shooter spectrum has now allowed us to directly compute the accretion luminosity by modeling of the Balmer continuum. We found that accretion makes up for only $0.1 \%$ of the bolometric luminosity of FU Tau A. Therefore, accretion luminosity cannot explain the overluminosity of FU Tau A. This is also corroborated by the absence of strong veiling in the $\mathrm{X}$-shooter spectrum.

Accretion possibly plays a role in another puzzling property of FU Tau A, its weak lithium absorption (see discussion in Sect. 6.3).

\subsubsection{Magnetic activity}

An alternative possibility for bringing FU Tau A in closer agreement with evolutionary models would be to shift the object horizontally towards higher effective temperature and mass. It was shown by Chabrier et al. (2007) and MacDonald \& Mullan (2009) that the influence of a strong magnetic field onto convection may provide such an effect. In this scenario FU Tau A appears cooler and has a larger radius than expected for its mass. Because $T_{\text {eff }}$ is obtained from observations without resorting to evolutionary models, while mass is model-dependent, this would imply a higher mass for FU Tau A than the $0.08 M_{\odot}$ obtained when extrapolating the object vertically down to the 1 Myr isochrone of Baraffe et al. (1998).

In Stelzer et al. (2010) we derived a mass of $\sim 0.2 M_{\odot}$ for FU Tau A assuming this scenario based on the stellar parameters given by Luhman et al. (2009a) and estimated the rotation period and the magnetic field strength using standard magnetospheric accretion models. These estimates can now be updated making use of the new values for the stellar parameters derived from the $\mathrm{X}$-shooter spectrum.

First, the new value for the bolometric luminosity corresponds to a mass of $0.15 M_{\odot}$ and a temperature of $3090 \mathrm{~K}$ on the 1 Myr isochrone of Baraffe et al. (1998). Our new value for the mass accretion rate (see Sect. 5.2.1) was based on a mass of $M_{*}=0.08 M_{\odot}$. This would be corrected downward to $\log \left\langle\dot{M}_{\text {acc }}\right\rangle\left[M_{\odot} / \mathrm{yr}\right]=-10.1 \pm 0.2$. With these new parameters and an inner disk truncation radius of $R_{\text {in }} \sim 2 \ldots .5 R_{*}$, the magnetic field strength predicted by Koenigl (1991) is $B \approx$ $40 \ldots 180 \mathrm{G}$. No magnetic field measurement has been performed yet for FU Tau Athat would allow these numbers to be tested. In any case, this estimate shows that the combination of stellar parameters and mass accretion rate observed for FU Tau A supports the presence of a strong surface field. The X-wind model (e.g., Mohanty \& Shu 2008), where the Keplerian angular velocity is equal to the stellar rotational velocity at the inner disk truncation radius, predicts a stellar rotation period of $P_{\text {rot }} \approx 1.4$...5.5 d for the range of $R_{\text {in }}$ given above. The value observed by Scholz et al. (2012) for the rotation period $\left(P_{\text {rot }}=4.0 \pm 0.2 \mathrm{~d}\right.$ ) is consistent with this prediction. As pointed out in our previous work this period is rather large for substellar mass according to the empirical trend between period and mass (e.g., Scholz \& Eislöffel 2005).

The influence of magnetic activity on the stellar parameters is also expected to have an effect on the lithium depletion of latetype stars, but this is unlikely to be relevant for FU Tau A as we describe in Sect. 6.3.

\subsubsection{Age}

Both FU Tau A and its brown dwarf companion FU Tau B have the highest absolute $J$ band magnitude of all Taurus members with which they share the effective temperatures $T_{\text {eff }} \sim 2800 \mathrm{~K}$ and $T_{\text {eff }} \sim 2400 \mathrm{~K}$, respectively (Scholz et al. 2012). This suggests that the pair is coeval but younger than the other VLM objects in Taurus. The low gravity of $\log g=3.5 \pm 0.5$ measured from the $\mathrm{X}$-shooter spectrum is a confirmation of the pre-main sequence nature of FU Tau A. However, in the standard picture extreme youth is not compatible with the weak lithium signature. Furthermore, there are no signs for an envelope that would be expected if the object were still in a protostellar phase.

\subsubsection{Binarity}

The picture could be further complicated if FU Tau A was a close binary. The maximum decrease in $L_{\text {bol }}$ obtained in this scenario is a factor two, which would make FU Tau A similar to some other VLM objects in Taurus that are overluminous with respect to the Baraffe et al. (1998) $1 \mathrm{Myr}$ isochrone. An equalmass binary with 140 AU separation $\left(\approx 1^{\prime \prime}\right.$ at the distance of Taurus) would have an RV amplitude of less than $\sim 1 \mathrm{~km} \mathrm{~s}^{-1}$ but a subarcsecond binary would produce a much greater RV signal. Binarity could be responsible for some of the difference between the RV we measured for FU Tau A and most Taurus members. One of the most obvious effects of binarity, besides its influence on the RV, would be that the rotational velocity has an unknown contribution from the orbital motion, and the measured $v \sin i$ is an upper limit. The $R \sin i$ estimate derived from the rotational velocity and the rotation period is, in fact, slightly higher than the stellar radius obtained from Stefan-Boltzmann's law $\left(R \sin i=1.6 \pm 0.5 R_{\odot}, R_{*}=1.4 R_{\odot}\right)$ but compatible with each other within the errors. The lithium equivalent width may also change in the case of binarity but in a way that is hard to predict without knowledge of the relative brightness of both binary components.

\subsubsection{Distance}

Finally, an obvious way to reduce the bolometric luminosity of FU Tau A would be a shorter distance. This would also lead to better agreement of the X-ray luminosity of FU Tau A with other objects of similar mass in Taurus (see Stelzer et al. 2010). For a shorter distance, the difference between the mean mass accretion rate obtained from the 20 emission lines and the mass accretion rate obtained from the $\mathrm{H} \alpha 10 \%$ width of FU Tau A could be reconciled, e.g. a distance of $75 \mathrm{pc}$ yields $\left\langle\log \dot{M}_{\mathrm{acc}}\right\rangle\left[M_{\odot} / \mathrm{yr}\right]=$ $-10.4 \pm 0.2$ for the mean of the emission lines with the stellar parameters from Sect. 3.2. The magnetic field would be larger $(B=90 \ldots 430 \mathrm{G})$ and the rotation faster $\left(P_{\text {rot }}=0.8 \ldots 2.9 \mathrm{~d}\right)$ than predicted for the magnetic activity scenario of Sect. 6.2.3. However, the theoretical predictions for these parameters should be taken at face value and not as firm prove of one or the other hypothesis.

The closer distance poses the problem of either the existence of a dark cloud apparently associated with the Taurus filaments but in reality located in the foreground or the unlikely projection of an isolated young binary onto a background dark cloud. An argument in favor of the latter case comes from the difference in the LSR velocity of FU Tau A $\left(12.5 \pm 2.9 \mathrm{~km} \mathrm{~s}^{-1}\right)$ and the value derived for Barnard 215 from CO maps $\left(v_{\mathrm{LSR}} \sim 7 \mathrm{~km} \mathrm{~s}^{-1}\right.$; Narayanan et al. 2008). On the other hand, the scenario where FU Tau A is in the foreground of Barnard 215, i.e. far from any signatures of star formation, raises the question of its origin. Ejection from a star forming site does not seem a viable option as for typical ejection speeds of $\sim 1 \mathrm{~km} \mathrm{~s}^{-1}$ (e.g. Bate et al. 2003) FU Tau A cannot have traveled beyond the (projected) diameter 
of Barnard 215 within its lifetime. Luhman et al. (2009a) mention that the SDSS image shows nebulosity that is centered on and surrounding FU Tau, which when combined with the signatures of youth, suggests that the object is associated with the dark cloud.

The RV of FU Tau A $\left(22.5 \pm 2.9 \mathrm{~km} \mathrm{~s}^{-1}\right)$ is marginally consistent with the RV distribution of Taurus, so is its $U V W$ space velocity $\left((U, V, W)=(-22.09,-9.08,-10.36) \mathrm{km} \mathrm{s}^{-1}\right)$. For the proper motion from Luhman et al. (2009a), a closer distance results in a space motion with a stronger deviation from the mean Taurus $U V W$ velocities (e.g., $(U, V, W)=(-21.80$, $-3.98,-8.64 \mathrm{~km} \mathrm{~s}^{-1}$ ) for $80 \mathrm{pc}$ ). However, considering the uncertainty of the proper motion inferred from the different literature sources, the change in space motion associated with a closer distance does not seem significant enough to allow us to draw conclusions.

\subsection{The lithium problem}

The lithium equivalent width measured in the $\mathrm{X}$-shooter spectrum of FU Tau A is $\sim 430 \mathrm{~m} \AA$. This corresponds to a lithium abundance below $\mathrm{A}(\mathrm{Li}) \sim 1.9$ according to the curves of growth calculated by Zapatero Osorio et al. (2002). At present, FU Tau A defines the lower envelope in the lithium equivalent widths of spectral types M6...M7. We have shown that this does not change even if weak veiling, consistent with the depth of the TiO absorption bands, is assumed. The second lowest value is represented by KPNO-Tau 5, another young BD in Taurus (Barrado y Navascués 2004). This object has no disk (Monin et al. 2010) and is below the canonical threshold for accretors in terms of its $\mathrm{H} \alpha$ equivalent width (Barrado y Navascués 2004), such that veiling seems unlikely to be present. Lithium measurements are available for a limited sample of such late-type young objects. Therefore, no conclusions can be drawn yet on the typical value for those spectral types. However, the large dispersion of lithium abundances is also seen at early-M types and has been noted as an unsolved problem.

Quite a number of parameters not taken into account in standard pre-main sequence evolutionary models may affect the lithium depletion timescale. Eggenberger et al. (2012) describe the influence of rotation and disk lifetime on the lithium depletion of solar-mass stars. Interestingly, rotation shifts the evolutionary tracks to slightly lower $T_{\text {eff }}$ in the HR diagram. Similarly, if the disks are longer-lived, lithium gets depleted more and more rapidly. The impact of rotation and disk lifetime on lithium depletion is at odds with the tendency for any given star's age derived from lithium to be older than the age obtained from its position in the HR diagram. Yee \& Jensen (2010) explain how the HR diagram ages and the lithium ages can be brought into qualitative agreement by the inflation of the radii due to the magnetic activity described in Sect. 6.2.3. The cooler effective temperatures associated with the larger radius would imply for a given star an older age from the HR diagram and a younger lithium depletion age. However, all these studies regard solartype stars, so their validity in the VLM regime cannot be taken for granted. Moreover, these arguments should break down for objects as young as FU Tau A, where no lithium depletion at all is expected from pre-main sequence models.

For FU Tau A another scenario is more plausible. Baraffe \& Chabrier (2010) argue that episodic accretion bursts during the pre-main sequence phase strongly decrease the timescale for lithium depletion in low-mass stars. In particular, they show that an accreting $0.1 M_{\odot}$ star may completely deplete its initial lithium content within $10 \mathrm{Myr}$ compared to >50 Myr required in the non-accreting case. For suitable values of the initial protostar mass and the strength and number of accretion outbursts, the observed lithium abundance of FU Tau A seems to agree with the predicted abundance from these models. However, in strong contrast to the observation for FU Tau A, including accretion in evolutionary models produces an underluminosity in the HR diagram (Baraffe et al. 2009). Special cases of these models evolve through a high-luminosity phase, but this phase is very shortlived, and the probability of observing its realization in nature is rather low. Moreover, FU Tau A is the only known VLM YSO with strong lithium underabundance that is a confirmed accretor.

A strong underabundance of lithium with respect to the cosmic abundance has also been found by Johnas et al. (2007), who modeled the high-resolution spectra of several young VLM objects in the Cha I star forming region with synthetic atmospheres. Their targets have spectral types M6/M7 and lithium abundances $\mathrm{A}(\mathrm{Li})<2.0$, just as FU Tau A. This seems to imply that our understanding of lithium depletion or line formation in VLM objects is incomplete.

\section{Summary and conclusions}

We have analyzed in detail an X-shooter spectrum of FU Tau A, and this has allowed us to redetermine its stellar parameters and provided a wealth of new information on activity, accretion, and outflow diagnostics. This allowed us to exclude accretion luminosity and extinction as the cause of the overluminosity of FU Tau A in the HR diagram. An inflated radius as a result of magnetic activity, leading to an underestimate of $T_{\text {eff }}$ and $M_{*}$, might be responsible for it. Strong activity is consistent with the high X-ray luminosity of FU Tau A. A combination of magnetic activity and very young age for the FU Tau binary may be responsible for its HR diagram location. Another possibility is a closer distance, but this is hard to explain due to the association of FU Tau A with the Barnard 215 dark cloud that is believed to be part of the Taurus complex. The possibility of FU Tau A being an unresolved binary must be taken into account. This would bring FU Tau A into better agreement with other luminous Taurus members and possibly with the RV distribution of Taurus. High-resolution spectroscopy is needed to search for a putative companion and to verify the unexpected evidence for lithium depletion. However, even with the available information, the startling properties of FU Tau A makes it a benchmark object for the further development of pre-main sequence evolutionary models.

Acknowledgements. J.M.A., B.S., and E.C. wish to thank G. Attusino for his help with the rapid completion of the manuscript. We appreciated the interesting discussions with $\mathrm{M}$. Bate and the fast response of the referee, K. Luhman. We thank the ESO staff for their support during the observations. We also appreciate the support of P. Goldoni, A. Modigliani, and G. Cupani in the use of the X-shooter pipeline. AS wishes to acknowledge funding by the Science Foundation Ireland through grant No. 10/RFP/AST2780.

\section{References}

Alcalá, J. M., Stelzer, B., Covino, E., et al. 2011, Astron. Nachr., 332, 242

Allard, F., Homeier, D., \& Freytag, B. 2012, in 16th Cambridge Workshop on Cool Stars, Stellar Systems, and the Sun, eds. C. M. Johns-Krull et al. (San Francisco: ASP), ASP Conf. Ser., 448, 91

Allers, K. N., Jaffe, D. T., Luhman, K. L., et al. 2007, ApJ, 657, 511

Bacciotti, F., \& Eislöffel, J. 1999, A\&A, 342, 717

Bacciotti, F., Whelan, E. T., Alcalá, J. M., et al. 2011, ApJ, 737, L26

Baraffe, I., \& Chabrier, G. 2010, A\&A, 521, A44

Baraffe, I., Chabrier, G., Allard, F., \& Hauschildt, P. H. 1998, A\&A, 337, 403

Baraffe, I., Chabrier, G., \& Gallardo, J. 2009, ApJ, 702, L27

Barrado y Navascués, D. 2004, A\&A, 422, 631

Bate, M. R., Bonnell, I. A., \& Bromm, V. 2003, MNRAS, 339, 577 
Bayo, A., Barrado, D., Stauffer, J., et al. 2011, A\&A, 536, A63 Beristain, G., Edwards, S., \& Kwan, J. 2001, ApJ, 551, 1037 Bertout, C., \& Genova, F. 2006, A\&A, 460, 499

Cabrit, S. 2009, Observational Constraints to Steady Jet Models in Young Stars, eds. K. Tsinganos, T. Ray, \& M. Stute, 247

Calvet, N., \& Gullbring, E. 1998, ApJ, 509, 802

Chabrier, G., Baraffe, I., Allard, F., \& Hauschildt, P. 2000, ApJ, 542, L119

Chabrier, G., Gallardo, J., \& Baraffe, I. 2007, A\&A, 472, L17

Costigan, G., Scholz, A., Stelzer, B., et al. 2012, MNRAS, 427, 1344

Dahn, C. C., Harris, H. C., Vrba, F. J., et al. 2002, AJ, 124, 1170

D’Antona, F., \& Mazzitelli, I. 1997, Mem. Soc. Astron. It., 68, 807

Ducourant, C., Teixeira, R., Périé, J. P., et al. 2005, A\&A, 438, 769

Eggenberger, P., Haemmerlé, L., Meynet, G., \& Maeder, A. 2012, A\&A, 539, A70

Gray, D. F. 1992, The observation and analysis of stellar photospheres (Cambridge University Press)

Gullbring, E., Hartmann, L., Briceno, C., \& Calvet, N. 1998, ApJ, 492, 323

Hartigan, P., Edwards, S., \& Ghandour, L. 1995, ApJ, 452, 736

Hartmann, L., Hewett, R., Stahler, S., \& Mathieu, R. D. 1986, ApJ, 309, 275

Hartmann, L., Hewett, R., \& Calvet, N. 1994, ApJ, 426, 669

Herczeg, G. J., \& Hillenbrand, L. A. 2008, ApJ, 681, 594

Jayawardhana, R., Mohanty, S., \& Basri, G. 2003, ApJ, 592, 282

Jeffries, R. D. 2006, Pre-Main-Sequence Lithium Depletion, eds. S. Randich, \& L. Pasquini, 163

Joergens, V., Kopytova, T., \& Pohl, A. 2012, A\&A, 548, A124

Johnas, C. M. S., Guenther, E. W., Joergens, V., Schweitzer, A., \& Hauschildt, P. H. 2007, A\&A, 475, 667

Kastner, J. H., Huenemoerder, D. P., Schulz, N. S., Canizares, C. R., \& Weintraub, D. A. 2002, ApJ, 567, 434

Koenigl, A. 1991, ApJ, 370, L39

Luhman, K. L. 1999, ApJ, 525, 466

Luhman, K. L., Mamajek, E. E., Allen, P. R., Muench, A. A., \& Finkbeiner, D. P. 2009a, ApJ, 691, 1265

Luhman, K. L., Mamajek, E. E., Allen, P. R., \& Cruz, K. L. 2009b, ApJ, 703, 399

Luhman, K. L., Mamajek, E. E., Allen, P. R., Muench, A. A., \& Finkbeiner, D. P. 2010, ApJ, 720, 1781
MacDonald, J., \& Mullan, D. J. 2009, ApJ, 700, 387

Manara, C. F., Testi, L., Rigliaco, E., et al. 2013, A\&A, 551, A107

Mentuch, E., Brandeker, A., van Kerkwijk, M. H., Jayawardhana, R., \& Hauschildt, P. H. 2008, ApJ, 689, 1127

Modigliani, A., Goldoni, P., Royer, F., et al. 2010, in SPIE Conf., 7737

Mohanty, S., \& Shu, F. H. 2008, ApJ, 687, 1323

Mohanty, S., Jayawardhana, R., \& Basri, G. 2005, ApJ, 626, 498

Monin, J.-L., Guieu, S., Pinte, C., et al. 2010, A\&A, 515, A91

Monin, J.-L., Whelan, E. T., Lefloch, B., Dougados, C., \& Alves de Oliveira, C. 2013, A\&A, 551, L1

Muzerolle, J., Calvet, N., \& Hartmann, L. 1998, ApJ, 492, 743

Narayanan, G., Heyer, M. H., Brunt, C., et al. 2008, ApJS, 177, 341

Natta, A., Testi, L., Muzerolle, J., et al. 2004, A\&A, 424, 603

Osterbrock, D. E. 1989, Astrophysics of gaseous nebulae and active galactic nuclei (Mill Valley, CA: University Science Books)

Riddick, F. C., Roche, P. F., \& Lucas, P. W. 2007, MNRAS, 381, 1067

Rigliaco, E., Natta, A., Testi, L., et al. 2012, A\&A, 548, A56

Rojas-Ayala, B., Covey, K. R., Muirhead, P. S., \& Lloyd, J. P. 2012, ApJ, 748, 93

Scholz, A., \& Eislöffel, J. 2005, A\&A, 429, 1007

Scholz, A., Stelzer, B., Costigan, G., et al. 2012, MNRAS, 419, 1271

Sestito, P., Palla, F., \& Randich, S. 2008, A\&A, 487, 965

Stelzer, B., Scholz, A., Argiroffi, C., \& Micela, G. 2010, MNRAS, 408, 1095

Torres, R. M., Loinard, L., Mioduszewski, A. J., \& Rodríguez, L. F. 2009, ApJ, 698,242

Valenti, J. A., Basri, G., \& Johns, C. M. 1993, AJ, 106, 2024

Valenti, J. A., Piskunov, N., \& Johns-Krull, C. M. 1998, ApJ, 498, 851

Walter, F. M., Brown, A., Mathieu, R. D., Myers, P. C., \& Vrba, F. J. 1988, AJ, 96, 297

Weingartner, J. C., \& Draine, B. T. 2001, ApJ, 548, 296

Whelan, E. T., Ray, T. P., Podio, L., Bacciotti, F., \& Randich, S. 2009, ApJ, 706, 1054

Whitworth, A., Bate, M. R., Nordlund, A., Reipurth, B., \& Zinnecker, H. 2007, Protostars and Planets V, 459

Yee, J. C., \& Jensen, E. L. N. 2010, ApJ, 711, 303

Zapatero Osorio, M. R., Béjar, V. J. S., Pavlenko, Y., et al. 2002, A\&A, 384, 937 